\title{
ESTIMULAÇÃO ELÉTRICA NEURAL PARA HIPERATIVIDADE VESICAL: UMA REVISÃO SISTEMÁTICA*
}

\author{
Daniela Minas Correia Fisioterapeuta, especialista em \\ uroginecologia, mestranda pela Escola \\ Bahiana de Medicina e Saúde Pública.
}

Juliana Menezes Santos Fisioterapeuta, especialista em

uroginecologia, mestranda pela Escola

Bahiana de Medicina e Saúde Pública.

Roseny Santos Ferreira $\begin{aligned} & \text { Fisioterapeuta, mestre em Família e } \\ & \text { sociedade contemporânea. }\end{aligned}$

$\begin{array}{ll}\text { Ubirajara Barroso Junior } & \begin{array}{l}\text { Urologista, doutor em medicina } \\ \text { (Urologia), professor adjunto da Escola } \\ \text { Bahiana de Medicina e Saúde Pública. }\end{array}\end{array}$

Patrícia Lordêlo Garboggini Fisioterapeuta, doutora em Medicina e Saúde Pública, professora adjunta da Escola Bahiana de Medicina e Saúde Pública.

Endereço para correspondência: pvslordêlo@htmail.com

\begin{abstract}
Resumo
Objetivo: Rever os estudos que utilizaram eletroestimulação no tratamento da hiperatividade vesical na infância. Metodologia: Trata-se de uma revisão sistemática. Os artigos foram pesquisados na base de dados Medline e na biblioteca de busca Cochrane (janeiro de 1990 a março de 2010), seguindo os critérios: 1) participantes com idades entre 0-17 anos; 2) participantes com diagnóstico de disfunção do trato urinário inferior do tipo hiperatividade vesical, não relacionados às anomalias congênitas ou doenças neurológicas; 3) idioma Inglês; 4) Tratamento pela estimulação elétrica neural. Resultados: Foram encontrados 11 artigos, sendo um randomizado Os artigos encontrados abordavam eletroestimulação percutânea no tibial posterior, estimulação elétrica neural (usar por extensoparassacral e associado a suprapúbica, além de dispositivos endoanal e anogenital. Os estudos demonstraram taxas de resolução, variando de 31 a $80 \%$ para a hiperatividade vesical, 25 a $84 \%$ e 13 a $84 \%$ para sintomas de urgência e incontinência, respectivamente. A recorrência da hiperatividade vesical variou de 10 a $25 \%$. Conclusão:Este estudo mostrou quealiteratura, naáreadeinteresse,é escassaeasevidênciasparaa eficácia dousodaestimulação elétrica neural na disfunção do trato urinário na infância é de difícil conclusão.
\end{abstract}

Palvavras-chave: Estimulação elétrica; Hiperatividade vesical; Disfunção miccional; Pediatria.

\section{ELECTRICAL NEURAL STIMULATION FOR OVERACTIVE BLADDER: SYSTEMATIC REVIEW}

\begin{abstract}
Objective: To review studies that used electrical stimulation in the treatment of overactive bladder in childhood. Methodology: This is a systematic review. Articles were searched in Medline and Cochrane library search (January 1990 to March 2010), the following criteria: 1) participants aged 0-17 years, 2) participants with a diagnosis of type DTUI hyperactivity bladder, not related to congenital abnormalities or neurological diseases, 3) English language, 4) treatment by the EEN. Results: We found 11 items. The articles found were related to posterior tibial percutaneous electrical stimulation, TENS parasacral and associated with suprapubic, and devices and endoanal acuminata, is a randomized study. The studies showed rates of resolution, ranging from 31 to $80 \%$ for overactive bladder, $25-84 \%$ and $13-84 \%$ for symptoms of urgency and incontinence, respectively. Recurrence of bladder hyperactivity ranged from 10 to $25 \%$. Conclusion: This study showed that the literature in the area of interest, is sparse and the evidence for the efficacy of neural electrical stimulation in urinary tract dysfunction in children is a difficult conclusion.
\end{abstract}

Keywords: Electrical stimulation; Overactive bladder; Voiding dysfunction. 


\section{INTRODUÇÃO}

A Disfunção do Trato Urinário Inferior (DTUI) é um distúrbio na fase de esvaziamento ou de enchimento do Trato Urinário Inferior (TUI) em crianças sem distúrbios neurológicos. ${ }^{(1)}$ A Hiperatividade Vesical (HV) é caracterizada por uma alteração da fase de enchimento vesical e sua principal apresentação clínica é de urgência miccional, podendo estarassociada também àincontinência e enurese. Além dos sintomas desagradáveis, a HV pode estar associada à Infecção do Trato Urinário (ITU), constipação, cicatrizes renais e problemas psicológicos. ${ }^{(1)}$

Classicamente, a HV tem sido tratada com antimuscarínicos. No entanto, alguns estudos têm relatado uma baixa taxa de resolução completa dos sintomas (cerca de $30 \%$ ) com este tipo de medicamento. ${ }^{(2-6)}$ Além disso, é frequente existirem efeitos colaterais como boca seca, constipação intestinal e intolerância ao calor. Os antimuscarínicos não são a solução ideal para a HV em crianças, por este motivo se faz necessário a busca por outras modalidades de tratamento, como a uroterapia que consiste em orientações comportamentais e miccionais, tais como ingestão hídrica adequada, micção de horário e não inibição do desejo miccional.

Em 1963, Caldwell descreveu a primeira experiência com a Estimulação Elétrica Neural (EEN) para o tratamento da incontinência urinária, utilizando eletrodos implantados peri-uretral, obteve resultados satisfatórios. No entanto, por ter sido realizado através de um procedimento cirúrgico, a aplicação clínica da EEN não se difundiu durante anos. ${ }^{(7)}$ Em 1968, Alexander e Rowan apresentaram alguns modelos de EEN com $66 \%$ de resultados desejáveis, ${ }^{(8)}$ no qual os eletrodos foram implantados.

Várias técnicas de EEN foram aplicadas ao longo dos tempos. Os eletrodos foram colocados nos genitais, ânus, intravesical, na área do nervo tibial posterior, transcutâneo ou implantado por um procedimento cirúrgico. ${ }^{(9-12)}$

Este trabalho tem como objetivo rever os estudos que utilizaram eletroestimulação no tratamento da hiperatividade vesical na infância, enfocando os resultados clínicos, técnicas de aplicação da EEN para a HV e sua taxa derecidiva.

\section{METODOLOGIA}

Trata-se de um trabalho de revisão sistemática. Os estudos foram pesquisados na base de dados Medline e na biblioteca de busca Cochrane (janeiro de 1990 a março de 
2010), usando os seguintes termos: electrical stimulation, neuromodulation, lower urinary tract dysfunction, bladder instability, overactive bladder, bladder dysfunction, bladder dysfunction, not neurogenic bladder, dysfunctional elimination syndrome (se a pesquisa foi feita exclusivamente em artigos da língua inglesa os teros devem estar em inglês aqui, conforme foram utilizados) A bibliografia dos ensaios obtidos e artigos de revisão também foram examinados.

O título e os resumos dos artigos identificados pela estratégia de busca foram avaliados com base nos seguintes critérios: 1) participantes com idades entre 0-17 anos; 2) participante com diagnóstico de DTUI do tipo hiperatividade vesical, não relacionado às anomalias congênitas ou doenças neurológicas; 3) idioma Inglês; 4) Tratamento pela EEN. Excluem-se os estudos que não diferenciavam o desfecho dos pacientes com DTUI daqueles com bexiga neurogênica, e aqueles que tiveram a associação com uma doença neuromuscular, por ser considerado como um possível fator de confusão, além das técnicas de eletroestimulação com eletrodos implantados, por se tratar de um procedimento cirúrgico.

Os artigos selecionados foram avaliados em relação aos resultados clínicos, técnicas de aplicação e taxa de recidivapara a HV (escolher entre BH ou HV e utilizar sempre o mesmo termo, sugiro hiperatividade vesical, conforme está no título ou alterar tudo desde o título) em crianças.

\section{RESULTADO}

\section{Técnica de EEN}

A Tabela 1 apresenta os dados referentes à aplicação das técnicas.

Existe uma grande variedade de técnicas de eletroestimulação no tratamento de hiperatividade vesical. Dos 11 artigos utilizados, foram encontrados estudos com eletroestimulação percutânea no tibial posterior, ${ }^{(13,11)}$ EEN parassacral ${ }^{(10,14-18)}$ ou associado à aplicaçãosuprapúbica, ${ }^{(19)}$ dispositivos endoanal ${ }^{(20)}$ e anogenital $^{(21)}$ a aplicação nestes dois casos não é igual? Anal? Não.

A duração do tratamento e o número de sessões por semana variaramentre os estudos. Para EEN parassacral, três estudos realizaram sessões diárias, totalizando 120 minutos por dia, por no mínino um mês ${ }^{(10,18,19)}$ e três estudos aplicaram por 20 minutos, 
3 vezes por semana, por no máximo 20 sessões. ${ }^{(15-17)}$ Em um estudo o tempo de aplicação também foi de 20 minutos, entretanto, com duas aplicações diárias. Para a estimulação percutânea no tibial posterior (EPTP), foi realizada uma sessão por semana, durante 12 semanas $^{(13)}$ ou por no máximo 20 sessões. $^{(11)}$ A EEN endoanal e anogenital foram realizadas diariamente de um a três meses. ${ }^{(20,21)}$

Os parâmetros elétricos utilizados também variaram. Na maioria dos estudos a intensidade da corrente foi aumentada até o nível tolerado pelo paciente. ${ }^{(11-21)}$ Em apenas um estudo a intensidade foi pré-determinada, variando de 0 a $10 \mathrm{~mA} .^{(13)}$

A largura de pulso variou de 150 a $1000 \mu$ s. Em quatro estudos não houve informações sobre a mesma. ${ }^{(11,15,19,21)}$ A freqüência da corrente variou de 2 a $150 \mathrm{~Hz}$. A maioria dos estudos usou uma corrente de baixa frequência, de $20 \mathrm{~Hz}$ ou menos. ${ }^{(10,13,15-}$ ${ }^{21)}$ Em apenas um estudo a frequência não foi declarada. ${ }^{(14)}$

Em sete estudos, a terapia farmacológica com anticolinérgico foi realizada antes da aplicação da eletroestimulação. ${ }^{(10,11,13,14,18,20,21)}$ Destes, quatro permaneceram com a terapia medicamentosa nas crianças que obtiveram alguma resposta a esse tipo tratamento. ${ }^{(13,14)}$ Um estudo utilizou anticolinérgico associado à antiespasmódicos. ${ }^{(11)}$ Em seis estudos, a eletroestimulação foi associada à uroterapia- descrever a uroterapia na introdução. ${ }^{(10,11,15-17,21)}$

\section{RESULTADOS CLÍNICOS}

A Tabela 2 mostra os resultados apresentados na literatura sobre a eficácia da EEN para $\mathrm{HV}$ em crianças. As taxas de resolução completa da HV e dos sintomas de Urgência e Incontinência (IU) foram de 31 a 80\%, 25 a 84\%, 13 a 84\% respectivamente. Quatro estudos utilizaram a escala visual analógica para demonstrar a melhora dos sintomas. $^{(15-17,19)}$

Hoebeke, Van Laecke, Everaert, Renson, De Paepe e Raes e Bower, Moore e Adams foram os primeiros a descrever a TENS para a HV em crianças. ${ }^{(10,19)}$ Hoebeke et al. ${ }^{(10)}$ utilizaram TENS parassacral e relataram resolução completa dos sintomas em $51 \%$ dos pacientes. A resolução da IU foi de $47 \%$ e $13 \%$ no estudo de Bower et al. e Malm-Buatsi, Nepple, Boyt, Austin e Cooper respectivamente. ${ }^{(16,19)}$ Nos estudos citados anteriormente, a EEN - aqui também escolher entre EEN e TENS e usar sempre o mesmo termo - foi usada na HV refratária a diversos tipos de tratamentos medicamentosos. 
Lordêlo, Teles, Veiga, Correia e Junior Barroso ${ }^{(17)}$ publicaram um ensaio clínico randomizado em que compararam a EEN parassacral com um grupo controle, no qual o estímulo foi aplicado sobre a área escapular.

Tabela 1 - Distribuição dos dados referente a aplicação da eletroestimulação: Tipo; frequência, largura de pulso, intensidade, tempo de aplicação e número de sessões

\begin{tabular}{|c|c|c|c|c|c|c|}
\hline Artigos & $\begin{array}{c}\text { Tipo de } \\
\text { Eletroestimula- } \\
\text { ção }\end{array}$ & $\begin{array}{c}\text { Frequên- } \\
\text { cia }(\mathbf{H z})\end{array}$ & $\begin{array}{c}\text { Largura de } \\
\text { Pulso } \\
(\mu \mathrm{s})\end{array}$ & $\begin{array}{c}\text { Intensida- } \\
\text { de } \\
(\mathrm{mA})\end{array}$ & $\begin{array}{l}\text { Tempo de } \\
\text { aplicação } \\
\text { (min) }\end{array}$ & $\begin{array}{l}\text { Número } \\
\text { de } \\
\text { sessões }\end{array}$ \\
\hline $\begin{array}{l}\text { De Gennaro } \\
\text { et al. }{ }^{13}\end{array}$ & EPTP & 20 & 200 & 0 a 10 & 30 & $\begin{array}{c}1 \mathrm{x} \\
\text { sempor } \\
12 \mathrm{sem}\end{array}$ \\
\hline $\begin{array}{l}\text { Hoebeke et } \\
\mathrm{al}^{14}\end{array}$ & EPTP & 20 & - & $\begin{array}{c}\text { Níveltolera } \\
\text { do }\end{array}$ & 30 & Max 20 \\
\hline $\begin{array}{l}\text { Hoebeke et } \\
\quad \mathrm{al}^{10}\end{array}$ & Parassacral & 2 & 150 & $\begin{array}{c}\text { Níveltolera } \\
\text { do }\end{array}$ & 120 & $\begin{array}{l}\text { Mín } 1 \\
\text { mês } \\
\text { 1x dia }\end{array}$ \\
\hline $\begin{array}{l}\text { Malm-Buatsi } \\
\quad \text { et al. }{ }^{14}\end{array}$ & Parassacral & - & 100 & $\begin{array}{c}\text { Níveltolera } \\
\text { do } \\
0 \text { a } 60\end{array}$ & 20 & $2 \mathrm{x}$ dia \\
\hline $\begin{array}{l}\text { Barroso et } \\
\text { al. }^{15}\end{array}$ & Parassacral & 10 & - & $\begin{array}{c}\text { Níveltolera } \\
\text { do (média } \\
22,2)\end{array}$ & 20 & Máx 20 \\
\hline $\begin{array}{l}\text { Lordelo et } \\
\text { al. }^{16}\end{array}$ & Parassacral & 10 & 700 & $\begin{array}{c}\text { Níveltolera } \\
\text { do }\end{array}$ & 20 & Máx 20 \\
\hline $\begin{array}{l}\text { Lordelo et } \\
\text { al. }^{17}\end{array}$ & Parassacral & 10 & 700 & $\begin{array}{c}\text { Níveltolera } \\
\text { do }\end{array}$ & 20 & 20 \\
\hline $\begin{array}{l}\text { Hagstroem et } \\
\mathrm{al}^{18}\end{array}$ & Parassacral & 10 & 200 & $\begin{array}{c}\text { Níveltolera } \\
\text { do } \\
\text { Até } 40\end{array}$ & 120 & $\begin{array}{l}4 \text { sem } \\
1 \mathrm{xdia}\end{array}$ \\
\hline$\underset{19}{\text { Bower et al }}$ & $\begin{array}{c}\text { Parasacral } \\
\text { esuprapubica }\end{array}$ & $\begin{array}{l}10 \text { (saida } \\
\text { sacral) e } \\
150 \\
\text { (suprapúb } \\
\text { ica) }\end{array}$ & - & $\begin{array}{c}\text { Níveltolera } \\
\text { do }\end{array}$ & 60 & $\begin{array}{l}\text { Mín } 1 \\
\text { mês } \\
2 \text { x dia }\end{array}$ \\
\hline $\begin{array}{l}\text { Trsinar and } \\
\text { Kralj }^{20}\end{array}$ & Endoanal & 20 & 1000 & $\begin{array}{l}\text { Níveltolera } \\
\text { do }\end{array}$ & 20 & $\begin{array}{c}1 \text { a } 2 \\
\text { meses }\end{array}$ \\
\hline Gladh et $\mathrm{al}^{21}$ & Anogenital & 10 & - & $\begin{array}{l}\text { Níveltolera } \\
\text { do }\end{array}$ & 20 & $\begin{array}{c}1 \text { ou } 2 x \\
\text { dia }\end{array}$ \\
\hline $\begin{array}{l}\text { EPTP:Eletro } \\
\text { estimulação } \\
\text { percutânea } \\
\text { no nervo } \\
\text { tibial } \\
\text { posterior }\end{array}$ & & & & & & \\
\hline
\end{tabular}

Lordêlo et al. ${ }^{(17)}$ referiram uma resolução completa dos sintomas em $62 \%$ das crianças do grupo teste e nenhum paciente no grupo controle. A medicação 
anticolinérgica foi utilizada apenas nos pacientes que não apresentaram resolução completa dos sintomas após a eletroestimulação.

Lordêlo, Soares, Maciel, Junior Macedo e Junior Barroso ${ }^{(16)}$ publicaram os resultados a longo prazo da EEN com um seguimento médio de 35 meses, variando de 6 a 80 meses. Para aqueles com mais de dois anos de seguimento $(n=30), 73 \%(n=22)$ tiveram melhora completa dos sintomas. ${ }^{(16)}$

O ensaio clínico randomizado realizado por Hagstroem et al., ${ }^{(18)}$ em pacientes com HV refratária, utilizando EEN parassacral, mostrou que $61 \%$ pacientes do grupo teste tiveram uma resposta na IU em comparação aos $17 \%$ do grupo controle.

Resultados promissores também têm sido relatados para a EPTP. O primeiro estudo sobre este tema foi publicado por Hoebeke, Renson, Petillon, Vande Walle, De Paepe $^{(11)}$ que relataram $80 \%$ de melhora dos sintomas da HVsemelhante ao estudo de DeGennaro, Capitanucci, Mastracci, Silveri, Gatti e Mosiello. ${ }^{(13)}$

Trsinar e Kralj utilizaram EEN com eletrodo endoanal, relatando resolução completa ou parcial da $\mathrm{HV}$ em $75 \%$ dos pacientes do grupo teste, entretanto não houve resposta completa dos sintomas no grupo controle, em que os pacientes tiveram os eletrodos endonais introduzidos, porém não ativados. ${ }^{(20)}$

\section{Taxa de recidiva}

A taxa de recorrência apresentou intervalo de $10-25 \%$ de acordo com os 5 estudos analisados (Tabela 2). 
Tabela 2 - Resultado de crianças com DTUI após eletroestimulação, em relação ao número de pacientes; resolução dos sintomas de urgência e incontinência; recidiva da hiperatividade vesical

\begin{tabular}{|c|c|c|c|c|c|}
\hline Artigos & Número de Pacientes & $\begin{array}{l}\text { Resolu- } \\
\text { ção da } \\
\text { HV }\end{array}$ & Urgência & Incontinência & Recidiva \\
\hline $\begin{array}{l}\text { De Gennaro } \\
\text { et al. }^{13}\end{array}$ & 10 & $80 \%$ & - & - & - \\
\hline $\begin{array}{l}\text { Hoebeke et } \\
\mathrm{al}^{11}\end{array}$ & 32 & $80 \%$ & $25 \%$ & $17 \%$ & - \\
\hline $\begin{array}{l}\text { Hoebeke et } \\
\mathrm{al}^{10}\end{array}$ & 41 & $51 \%$ & - & - & $25 \%$ \\
\hline $\begin{array}{l}\text { Malm-Buatsi } \\
\text { et al. }^{18}\end{array}$ & 18 & - & - & $13 \%$ & - \\
\hline $\begin{array}{l}\text { Barroso et } \\
\text { al. }^{15}\end{array}$ & 19 & $63 \%$ & $79 \%$ & $75 \%$ & - \\
\hline $\begin{array}{l}\text { Lordelo et } \\
\text { al. }^{16}\end{array}$ & 49 & $78 \%$ & $84 \%$ & $74 \%$ & $10 \%$ \\
\hline $\begin{array}{l}\text { Lordelo et } \\
\text { al. }^{17}\end{array}$ & $\begin{array}{l}21 \text { (teste) e } 16 \\
\text { (controle) }\end{array}$ & $62 \%$ & - & $84 \%$ & $11 \%$ \\
\hline $\begin{array}{l}\text { Hagstroem et } \\
\mathrm{al}^{18}\end{array}$ & $\begin{array}{c}13 \text { (teste) e } 12 \\
\text { (controle) }\end{array}$ & - & - & $61 \%$ & - \\
\hline Bower et al ${ }^{1 y}$ & 17 & - & - & $47 \%$ & - \\
\hline$\underset{\text { Kralj }^{20}}{\text { Trsinar and }}$ & $\begin{array}{l}73 \text { (teste) e } \\
21 \text { (controle) }\end{array}$ & $31 \%$ & - & - & - \\
\hline Gladh et $\mathrm{al}^{21}$ & 48 & $38 \%$ & - & - & - \\
\hline
\end{tabular}

\section{DISCUSSÃO}

A literatura identificada nesta revisão, a respeito da técnica de eletroestimulação e resposta clínica no tratamento da DTUI em crianças, é escassa.

A utilização de nomenclaturas não estabelecidas pela padronização da International Children's Continence Society (ICCS) torna difícil a comparação dos resultados encontrados. ${ }^{(1)}$ A existência da incontinência urinária e urgência miccional, sua gravidade, além da sua frequência não foram analisadas na maioria dos estudos.

A aplicação das diversas técnicas e o fato dos parâmetros não serem reportados em todos os estudos ${ }^{(10,11,14,15,19,21)}$ dificultam a comparação dos métodos, evidenciando a necessidade de ensaios clínicos, assim como a comparação com os atuais parâmetros existentes. Isso requer profissionais treinados no uso da terapêutica ao invés de utilizarem os parâmetros previamente estabelecidos pelos fabricantes.

Lordêlo et al. ${ }^{(17)}$ referem que a eletroestimulação deve ser realizada associada a uroterapia, que consiste em orientações comportamentais, incluindo ingesta adequada 
de líquido durante o dia e a micção com horários regulares, contudo, infelizmente, não existem estudos prospectivos controlados disponíveis sobre a utilização de uroterapia para o tratamento da HV.

A avaliação dos resultados foi variável entre os estudos. A maioria avaliou taxa de sucesso subjetivamente. ${ }^{(15-17,20)}$ Perguntar aos pais se a criança melhorou dos sintomas, parcial ou totalmente, após o procedimento, não é a melhor maneira de avaliar o resultado. Apesar de ser uma ferramenta valiosa, é muito subjetiva e possui importante viés do entrevistador e dos sujeitos.

Uma avaliação mais objetiva seria a aplicação do diário miccional em que os pais tomariam nota do número de micções, média e máxima de volume urinado e presença de manobras de contenção, episódios de urgência miccional e incontinência urinária durante semanas.

\section{CONCLUSÕES}

Este estudo mostrou que a literatura na área de interesse é escassa e as evidências para a eficácia do uso da EEN na DTUI na infância são de difícil conclusão, o que requer mais investigação para elucidar os parâmetros ótimos e as crianças para as quais o tratamento é mais útil, entretanto vale salientar que foi observado nos estudos uma tendência de melhora/cura com esse tipo de tratamento, ainda que seus parâmetros ideais não tenham sido padronizados.

Pesquisas futuras devem se concentrar em entender qual é a melhor frequência e intensidade, bem como a importância da largura de pulso. Também se faz necessária uma comparação com diferentes métodos de EEN.

\section{REFERÊNCIAS}

1. Neveus T, von GA, Hoebeke P, Hjalmas K, Bauer S, Bower W et al. The standardization of terminology of lower urinary tract function in children and adolescents: report from the Standardisation Committee of the International Children's Continence Society. J. urol. 2006;176(1):314-24.

2. Varlam DE, Dippell J. Non-neurogenic bladder and chronic renal insufficiency in childhood. Pediatr. nephrol. 1995;9(1):1-5 
3. Garat Barredo JM, Caffaratti SJ, de la Pena E. [Treatment of bladder instability (nonneurogenic hyperactive bladder) in children, with tolterodine]. Actas urol. esp 2004;28(2):122-8.

4. Raes A, Hoebeke P, Segaert I, Van LE, Dehoorne J, Vande Walle J. Retrospective analysis of efficacy and tolerability of tolterodine in children with overactive bladder. Eur urol. 2004;45(2):240-4

5. Reinberg Y, Crocker J, Wolpert J, Vandersteen D. Therapeutic efficacy of extended release oxybutynin chloride, and immediate release and long acting tolterodine tartrate in children with diurnal urinary incontinence. J. urol. 2003;169(1):317-9

6. Van Arendonk KJ, Austin JC, Boyt MA, Cooper CS. Frequency of wetting is predictive of response to anticholinergic treatment in children with overactive bladder. Urology. 2006;67(5):1049-53

7. Caldwell KP. The electrical control of sphincter incompetence. Lancet. 1963;2(7300):174-5.

8. Alexander S, Rowan D. An electric pessary for stress incontinence. Lancet. 1968;1(7545):728.

9. Junior Barroso U, Lordelo P, Lopes AA, Andrade J, Junior Macedo A, Ortiz V. Nonpharmacological treatment of lower urinary tract dysfunction using biofeedback and transcutaneous electrical stimulation: a pilot study. BJU Int. 2006;98(1):166-71.

10. Hoebeke P, Van Laecke E, Everaert K, Renson C, De Paepe H, Raes A et al. Transcutaneous neuromodulation for the urge syndrome in children: a pilot study. J. urol. 2001;166:2416-9.

11. Hoebeke P, Renson C, Petillon L, Vande Walle J, De Paepe H. Percutaneous electrical nerve stimulation in children with therapy resistant nonneuropathic bladder sphincter dysfunction: a pilot study. J. urol. 2002;168(6):2605-7.

12. Tanagho EA, Schmidt RA. Bladder pacemaker: scientific basis and clinical future. Urology. 1982;20(6):614-9.

13. De Gennaro M, Capitanucci ML, Mastracci P, Silveri M, Gatti C, G Mosiello. Percutaneoustibial nerve neuromodulation is well tolerated in children and effective for treating refractory vesical dysfunction. J urol. 2004;171:1911-3.

14. Malm-Buatsi E, Nepple KG, Boyt MA, Austin JC, Cooper CS. Efficacy of transcutaneous electrical nerve stimulation in children with $\mathrm{OAB}$ refractory to pharmacotherapy. Urology 2007;70(5):980-3.

15. Junior Barroso U, Lordêlo P, Lopes AA, Andrade J, Junior Macedo A, Ortiz V. Nonpharmacological treatment of lower urinary tract dysfunction using biofeedback and transcutaneous electrical stimulation: a pilot study. BJU Int. 2006;98:166-71. 
16. Lordêlo P, Soares PV, Maciel I, Junior Macedo A, Junior Barroso U. Prospective study of transcutaneous parasacral electrical stimulation for overactive bladder in children: long-term results. J. urol. 2009;182:2900-4.

17. Lordêlo P, Teles A, Veiga ML, Correia LC, Junior Barroso U. Transcutaneous Electrical Nerve Stimulation in Children with Overactive Bladders: A Randomized Clinical Trial. J. urol. 2010, 184:683-9.

18. Hagstroem S, Mahler B, Madsen B, et al: Transcutaneous electrical nerve stimulation for refractory daytime urinary urge incontinence. J. urol. 2009;182:2072-8.

19. Bower WF, Moore KH, Adams. RD: A pilot study of the home application of transcutaneous neuromodulation in children with urgency or urge incontinence. J. urol. $2001 ; 166: 2420-2$.

20. Trsinar B, Kraij B: Maximal electrical stimulation in children with unstable bladder and nocturnal enuresis and/or daytime incontinence: a controlled study. Neurourol urodyn. 1996;15:133-42.

21. Gladh G, Mattsson S, Lindström S. Anogenital electrical stimulation as treatment of urge incontinence in children. BJU Int. 2001;87:366-71. 